\title{
An Alternative Benchmarking Approach for Electricity Utility Regulation Using Maximum Entropy*
}

\author{
Elvira Silva $^{1} \quad$ Pedro Macedo $^{2} \quad$ Isabel Soares $^{1}$ \\ ${ }^{1}$ Faculty of Economics \& Center for Economics and Finance at University of Porto (CEF.UP), Porto, Portugal \\ ${ }^{2}$ CIDMA - Center for Research and Development in Mathematics and Applications, \\ Department of Mathematics, University of Aveiro, Aveiro, Portugal
}

The main purpose of this study is to present an alternative benchmarking approach that can be used by national regulators of utilities. It is widely known that the lack of sizeable data sets limits the choice of the benchmarking method and the specification of the model to set price controls within incentive-based regulation. Ill-posed frontier models are the problem that some national regulators have been facing. Maximum entropy estimators are useful in the estimation of such ill-posed models, in particular in models exhibiting small sample sizes, collinearity and nonnormal errors, as well as in models where the number of parameters to be estimated exceeds the number of observations available. The empirical study involves a sample data used by the Portuguese regulator of the electricity sector to set the parameters for the electricity distribution companies in the regulatory period of 20122014. DEA and maximum entropy methods are applied and the efficiency results are compared.

Keywords: DEA; Electricity distribution; Maximum Entropy; Regulation; Technical efficiency.

\section{Introduction}

The evidence from the literature on incentive-based regulation in the electricity sector indicates that the size of a country's electricity sector (i.e., the number of companies in the electricity value chain) tends to influence the choice of benchmarking methods as well as the specification of the frontier model. The size of a country's electricity sector and the problems with (or lack of) data are among the reasons pointed out by national regulators who do not employ benchmarking techniques [7]. Countries with a small number of transmission and distribution companies are constrained in the choice of methods, because the limited number of similar companies is a barrier to the use of frontier benchmarking methods; e.g., [6] and [13].

*Final version of this work is published in the Proceedings of the $13^{\text {th }}$ International Conference on the European Energy Market (EEM), 2016,
IEEE (DOI: 10.1109/EEM.2016.7521250; http://ieeexplore.ieee.org/document/7521250/?arnumber=7521250).

(C) 2016 IEEE. Personal use of this material is permitted. Permission from IEEE must be obtained for all other uses, in any current or future media, including reprinting/republishing this material for advertising or promotional purposes, creating new collective works, for resale or redistribution to servers or lists, or reuse of any copyrighted component of this work in other works. 
The majority of energy regulators who employ benchmarking techniques are countries with a larger number of regulated companies (e.g., Finland, Britain and Germany). There are, however, several countries with very few regulated companies (e.g., Portugal, Slovenia and Panama) that employ benchmarking techniques using international data. The use of international data can improve the options, yet there are still advances to be made in the availability and use of international data [6]. Even using international benchmarking analysis, the size of the sample may not be enough, due to the limited number of suitable comparators that can be identified, to allow the use of certain benchmarking methods. Furthermore, an additional problem with the use of international data is the difficulty of comparing and applying data to different country contexts.

The main purpose of this study is to present an alternative benchmarking approach based on the maximum entropy (ME) principle, namely the generalized maximum entropy (GME) estimator, to set price controls within incentive-based regulation. The data sample used in this study is the data on electricity distribution companies used by the Portuguese regulator of the electricity sector (ERSE) to set the parameters for the distribution companies in the regulatory period of 2012-2014 [2]. DEA and GME are applied to the ERSE data set and the efficiency results are compared.

\section{Brief Literature Review}

The most common benchmarking methods used in the electricity sector are econometric modeling, involving constrained ordinary least squares (COLS) and stochastic frontier analysis (SFA) with maximum likelihood (ML), indexing (e.g., unit costs and TFP indexes), and mathematical modeling using DEA (e.g., [11]). There are several studies comparing the performance of several benchmarking methods in the context of regulating the electricity sector or/and using a Monte Carlo simulation study. Reference [8] discuss the effect of the choice of the benchmarking methods using DEA, COLS and SFA models applied to an international sample of 63 regional electricity distribution utilities in six European countries. One of the results of this study is that the selection of benchmarking methods, model specification, and variables (choice of inputs, outputs and environmental variables) can affect the efficiency scores, as well as the rank order of firms. Consequently, the robustness and accuracy of calculated $X$-factors can be questioned. Also, frontier approaches are sensitive to shocks and errors in the data. This is particularly the case when cross-sectional data is used and benchmarking methods that do not consider stochastic error terms as the DEA and COLS [8], [9].

\section{Distance Function and Estimation}

The radial input distance function is developed by [16]. For an overview of this function and its properties, please see [3]. It is defined by

$$
D(y, x)=\sup _{\beta}\{\beta>0: x / \beta \in V(y)\}, \forall y \in \Re_{+}^{M},
$$

where $x$ is a $N$-input vector, $y$ is a $M$-output vector and $V(y)$ is the input (requirement) set for $y$. By definition, $D(y, x) \geq 1 \Leftrightarrow x \in V(y)$. The DEA model, assuming constant returns to scale (CRS), is given as

$$
\begin{aligned}
T \hat{E}(y, x \mid C) & =(1 / \hat{D}(y, x \mid C))=\min _{\lambda, z}\left\{\lambda: \lambda x \in \hat{V}_{J}(y \mid C)\right\} \\
& =\min _{\lambda, z}\left\{\lambda: \sum_{j=1}^{J} z^{j} y_{m j} \geq y_{m i}, \quad m=1, \ldots, M ; \sum_{j=1}^{J} z^{j} x_{n j} \leq \lambda x_{n i}, \quad n=1, \ldots, N ; z^{j} \geq 0, j=1, \ldots, J\right\} .
\end{aligned}
$$


Under the hypothesis of variable returns to scale (VRS), the DEA model is similar to (2) by adding the constraint $\sum_{j} z^{j}=1$.

ME estimation requires the specification of a parametric functional form for the radial input distance function. The distance function is specified as a translog. Choosing input $x_{l}$ and imposing homogeneity of degree 1 in the inputs, the distance function is given as

$$
\begin{aligned}
-\ln x_{1 i}= & \alpha_{0 i}+\sum_{m=1}^{M} \alpha_{m i} \ln y_{m i}+\sum_{n=2}^{N} \beta_{n i} \ln x_{n i}^{*}+\frac{1}{2} \sum_{m=1}^{M} \sum_{l=1}^{M} \alpha_{m l i} \ln y_{m i} \ln y_{l i} \\
& +\frac{1}{2} \sum_{n=2}^{N} \sum_{k=2}^{N} \beta_{n k i} \ln x_{n i}^{*} \ln x_{k i}^{*}+\frac{1}{2} \sum_{m=1}^{M} \sum_{n=2}^{N} \gamma_{m n i} \ln y_{m i} \ln x_{n i}^{*}+\varepsilon_{i}
\end{aligned}
$$

where $x_{n i}^{*}=x_{n i} / x_{1 i}$ and $\varepsilon_{i}=v_{i}-u_{i}$. See [10] for further details on stochastic distance functions.

The ME estimation, by avoiding criticisms and difficulties of DEA and SFA, appears to be a promising approach in efficiency analysis; e.g., [1], [12], [14] and [15]. Briefly, the GME estimator (proposed by [5]) extended to the SFA context is given by

$$
\underset{p, w, \rho}{\arg \max }\left\{-p^{\prime} \ln p-w^{\prime} \ln w-\rho^{\prime} \ln p\right\}
$$

subject to the model constraint,

and the three additivity constraints,

$$
\ln y=X Z p+A w-B \rho,
$$

$$
\begin{aligned}
& 1_{K}=\left(I_{K} \otimes 1_{M}^{\prime}\right) p, \\
& 1_{N}=\left(I_{N} \otimes 1_{J}^{\prime}\right) w, \\
& 1_{N}=\left(I_{N} \otimes 1_{L}^{\prime}\right) \rho,
\end{aligned}
$$

where $\otimes$ represents the Kronecker product. Additional details on the support matrices are provided next.

\section{Data and Empirical Results}

The real data used in this study is the data sample used by ERSE to set the parameters for the electricity distribution companies in the regulatory period of 2012-2014. Before discussing the data, the electricity distribution activity in Portugal is characterized briefly as well as the methodology employed by ERSE. The EDP distribution company is almost a monopolist in mainland Portugal assuring the distribution of electricity to $99 \%$ of the total population. High voltage and medium voltage electricity distribution is exclusively assured by EDP distribution; while the low voltage distribution is almost assured by EDP. The remaining consumers are served by ten small regional companies. There are two other small distribution companies, one in Azores (EDA) and one in Madeira (EEM).

ERSE employs an international data set comprising European distribution utilities with available information and suitable comparators with respect to OPEX and the main cost drivers. The main cost drivers are the number of customers, energy delivered $(\mathrm{GWh})$ and the network length $(\mathrm{Km})$. OPEX are controllable costs by the companies, calculated on the basis of 2009 constant prices. In the case of ESB (Ireland) and SP distribution (UK) whose year of the data is not 2009, OPEX is calculated on the basis of the inflation rate of those countries. In order to make the data among countries comparable, OPEX is calculated in US\$ PPP. For further details on the data set, please see [2]. 
Regarding the model specification, four models are considered specifying different returns to scale and variables specification. The four models are

Model 1: CRS, $\mathrm{x}_{1}=$ OPEX, $\mathrm{y}_{1}=$ energy delivered, $\mathrm{y}_{2}=$ number of customers, $\mathrm{y}_{3}=$ network length.

Model 2: CRS, $\mathrm{x}_{1}=$ OPEX, $\mathrm{x}_{2}=$ network length, $\mathrm{y}_{1}=$ energy delivered, $\mathrm{y}_{2}=$ number of customers.

Model 3: VRS, $x_{1}=$ OPEX, $y_{1}=$ energy delivered, $y_{2}=$ number of customers, $y_{3}=$ network length.

Model 4: VRS, $x_{1}=$ OPEX, $x_{2}=$ network length, $y_{1}=$ energy delivered, $y_{2}=$ number of customers.

Network length is not an obvious measure of output since it measures a capital input. The rationale for considering it as an output is that it measures the difficulty of topology [13]. In models 1 and 3, network length is defined as an output; in models 2 and 4, network length is a fixed input.

Due to the extremely small size of the sample (eleven observations), it is not possible to use COLS and ML. DEA and SFA with GME are employed in this study. DEA efficiency scores are reported in Table 1. As expected, the DEA efficiency scores under VRS (models 3 and 4) are greater than or equal to the ones generated under CRS (models 1 and 2). The sensitivity of the efficiency scores is high to the specification of the network length as an output variable or a fixed input variable. The efficiency scores, under the hypothesis of CRS, decrease for nine companies and remain constant for the other two companies when the network length changes from an output variable to a fixed input. However, under VRS, the efficiency scores remain constant for six companies, decreases for three of them and increases for the remaining two. East and NEDL are fully efficient in every DEA model. The rankings of the companies change, in general, across model. For example, the ranking of EDP is: $1^{\text {st }}$ in model $3,2^{\text {nd }}$ in models 1 and 4 , and $5^{\text {th }}$ in model 2 . Sibelga is the least efficient company in models 1, 2 and 3 and fully efficient in model 4.

Our results are consistent with the findings in some previous studies (e.g., [4] and [8]). "Our experience in Europe shows, however, that different versions of a DEA model will give quite different results and that there is no way to tell which set of results is most reliable.(...)" [17, p. 45].

Table 1: DEA efficiency scores

\begin{tabular}{|l|c|c|c|c|}
\hline Company & Model 1 & Model 2 & Model 3 & Model 4 \\
\hline East & 1.0000 & 1.0000 & 1.0000 & 1.0000 \\
\hline EDP & 0.9094 & 0.6035 & 1.0000 & 0.9113 \\
\hline Endesa & 0.4354 & 0.3603 & 1.0000 & 1.0000 \\
\hline Enel Distribuzione & 0.4934 & 0.3197 & 1.0000 & 1.0000 \\
\hline ESB & 0.6173 & 0.2085 & 0.6708 & 0.2216 \\
\hline NEDL & 1.0000 & 1.0000 & 1.0000 & 1.0000 \\
\hline PPC & 0.8033 & 0.6467 & 1.0000 & 1.0000 \\
\hline Sibelga & 0.1897 & 0.1772 & 0.6054 & 1.0000 \\
\hline South East & 0.8339 & 0.7425 & 0.8691 & 0.8830 \\
\hline SP Distribution & 1.0000 & 0.8578 & 1.0000 & 1.0000 \\
\hline V̈̈chodoslovenská & 0.3474 & 0.2132 & 0.6229 & 0.5597 \\
\hline
\end{tabular}

Considering now the results from SFA with the GME estimator, and before the discussion of the estimation procedures, it is important to note that these are ill-posed models because the ratio of the largest singular value of the design matrices with the smallest singular value is very high in all of them. Additionally, the number of parameters to estimate exceeds the number of observations available in some models. Thus, given these characteristics, the use of traditional estimation techniques in SFA should be avoided and ME estimators are recommended. 
The support matrices $Z$ and $A$ are defined by the researcher based on prior information. In this work, the supports in $Z$ are defined through $[-10,10]$ for all the parameters of the models and the supports in the matrix $A$ are defined by the 3-sigma rule considering the standard deviation of the noisy observations. The supports in matrix $B$ are established accordingly to [12], where the upper bound of each support is given by $-\ln \left(D E A_{n}\right)$, being $D E A_{n}$ the lower efficiency estimate obtained by DEA in each sample. Five points in the supports $(M=J=L=5)$ of each support matrix are considered. SFA efficiency scores are reported in Table 2.

Table 2: SFA with GME efficiency scores

\begin{tabular}{|l|c|c|c|c|}
\hline Company & Model 1 & Model 2 & Model 3 & Model 4 \\
\hline East & 0.7515 & 0.8044 & 0.8986 & 0.7512 \\
\hline EDP & 0.7477 & 0.7104 & 0.8948 & 0.7457 \\
\hline Endesa & 0.6564 & 0.6449 & 0.8900 & 0.6820 \\
\hline Enel Distribuzione & 0.6434 & 0.5904 & 0.8915 & 0.7341 \\
\hline ESB & 0.7401 & 0.5831 & 0.8978 & 0.7413 \\
\hline NEDL & 0.6849 & 0.8262 & 0.8918 & 0.7263 \\
\hline PPC & 0.7242 & 0.7148 & 0.8917 & 0.7218 \\
\hline Sibelga & 0.6352 & 0.5701 & 0.8872 & 0.7010 \\
\hline South East & 0.7415 & 0.7756 & 0.8972 & 0.7401 \\
\hline SP Distribution & 0.7866 & 0.7849 & 0.9023 & 0.7764 \\
\hline Vÿchodoslovenská & 0.6562 & 0.5961 & 0.8903 & 0.7046 \\
\hline
\end{tabular}

As verified with DEA, the mean values of SFA efficiency scores under VRS (models 3 and 4) are greater than the ones generated under CRS (models 1 and 2). Moreover, the efficiency scores are sensitive to the specification of the network length as an output variable or a fixed input variable. Although the rankings change across models, there are some similarities at the top and at the bottom of them: for example, SP Distribution is the most efficient company in models 1, 3 and 4, and the third in model 2; East is the second company in the four models; Sibelga is the least efficient company in models 1, 2 and 3, and the second least efficient in model 4 . The rankings of EDP are worse under the SFA approach: $3^{\text {rd }}$ in models 1 and $4,5^{\text {th }}$ in model 3 and $6^{\text {th }}$ in model 2.

\section{Conclusions}

The main purpose of this study is to propose an alternative benchmarking approach that can be used by national regulators of utilities. The lack of sizeable data sets limits the choice of the benchmarking method as well as the specification of the model to set price controls within incentive-based regulation. National regulators have been facing a problem of ill-posed frontier models. Maximum entropy estimators are useful in the estimation of such illposed models, in particular in models exhibiting small sample sizes, collinearity and non-normal errors, as well as in models where the number of parameters to be estimated exceeds the number of observations available.

The empirical study involves a sample data used by the Portuguese regulator of the electricity sector to set the parameters for the electricity distribution companies in the regulatory period of 2010-2014. DEA and ME estimates of technical efficiency are compared.

The curse of dimensionality is evident from the results of the DEA models 3 and 4 where approximately $64 \%$ of the distribution utilities are fully efficient. Furthermore, models 1 and 2 give quite different results from models 3 and 4. For example, Endesa, Enel Distribuizone and Sibelga are very inefficient companies according to models 1 and 2 and become fully efficient when models 3 and 4 are employed. 
Considering the SFA with the GME estimator, note that these models are ill-posed because the ratio of the largest singular value of the design matrices with the smallest singular value is very high in all of them. Additionally, the number of parameters to be estimated is greater than the number of observations. The GME results indicate that no distribution utility is fully efficient. Although the rankings of the electricity distribution companies change across models, there are some similarities at the top and at the bottom of them.

\section{Acknowledgements}

This work was supported in part by the Portuguese Foundation for Science and Technology (FCT - Fundação para a Ciência e a Tecnologia), through CIDMA - Center for Research and Development in Mathematics and Applications, within project UID/MAT/04106/2013.

\section{References}

[1] R. Campbell, K. Rogers, and J. Rezek, "Efficient frontier estimation: a maximum entropy approach,” Journal of Productivity Analysis, vol. 30, pp. 213-221, 2008.

[2] ERSE, "Parâmetros de regulação para o período 2011 a 2014," Lisboa, 2011.

[3] R. Färe and D. Primont, Multi-Output Production and Duality: Theory and Applications, New York: Kluwer Academic Publishers, 1995.

[4] M. Farsi and M. Filippini, "Regulation and measuring cost-efficiency with panel data models: Application to electricity distribution utilities," Review of Industrial Organization, vol. 25, pp. 1-19, 2004.

[5] A. Golan, G. Judge, and D. Miller, Maximum entropy econometrics: Robust estimation with limited data, Chischester: John Wiley \& Sons, 1996.

[6] A. B. Haney and M. G. Pollitt, "Exploring the determinants of "best practice" benchmarking in electricity network regulation," Energy Policy, vol. 39, pp. 7739-7746, 2011.

[7] A. B. Haney and M. G. Pollitt, "Efficiency analysis of energy networks: An international survey of regulators," Energy Policy, vol. 37, pp. 5814-5830, 2009.

[8] T. Jasmab and M. Pollitt, "International benchmarking and regulation: an application to European electricity distribution utilities," Energy Policy, vol. 31, pp. 1609-1622, 2003.

[9] T. Jasmab and M. Pollitt, "Benchmarking and regulation: international electricity experience," Utilities Policy, vol. 9, pp. 107-130, 2001.

[10] S. C. Kumbhakar and C. A. K. Lovell, Stochastic Frontier Analysis, Cambridge: Cambridge University Press, 2000.

[11] M. N. Lowry and L. Getachew, "Statistical benchmarking in utility regulation: role, standards and methods," Energy Policy, vol. 37, pp. 1323-1330, 2009.

[12] P. Macedo, E. Silva, and M. Scotto, "Technical efficiency with state-contingent production frontiers using maximum entropy estimators," Journal of Productivity Analysis, vol. 41, pp. 131-140, 2014.

[13] M. Pollitt, "The role of efficiency estimates in regulatory price reviews: Ofgem's approach to benchmarking electricity networks," Utilities Policy, vol. 13, pp. 279-288, 2005.

[14] J. P. Rezek, R. C. Campbell, and K. E. Rogers, "Assessing total factor productivity growth in Sub-Saharan African agriculture," Journal of Agricultural Economics, vol. 62, pp. 357-374, 2011.

[15] M. Robaina-Alves, V. Moutinho, and P. Macedo, "A new frontier approach to model the eco-efficiency in European countries," Journal of Cleaner Production, vol. 103, pp. 562-573, 2015.

[16] R. W. Shephard, Cost and Production Functions, Princeton: Princeton University Press, 1953. 
[17] G. Shuttleworth, "Firm-specific productive efficiency: A response," The Electricity Journal, vol. 16, pp. 42-50, 2003. 